(c) 2019 Universidad Nacional Autónoma de México, Facultad de Estudios Superiores Zaragoza.

Este es un artículo Open Access bajo la licencia CC BY-NC-ND (http://creativecommons.org/licenses/by-nc-nd/4.0/).

TIP Revista Especializada en Ciencias Químico-Biológicas, 22: 1-12, 2019.

DOI: 10.22201/fesz.23958723e.2019.0.196

\title{
Estructura y función de las oxigenasas tipo Rieske/mononuclear
}

Javier Carrillo-Campos Departamento de Bioquímica, Facultad de Química, Ciudad Universitaria Universidad Nacional Autónoma de México, Alcaldía de Coyoacán 04510, Ciudad de México, México. E-mail: jc890917@hotmail.com

\section{RESUMEN}

Las oxigenasas Rieske/mononuclear son un grupo de metaloenzimas que catalizan la oxidación de una variedad de compuestos, destaca su participación en la degradación de compuestos xenobióticos contaminantes; estas enzimas también participan en la biosíntesis de algunos compuestos de interés comercial. Poseen una amplia especificidad por el sustrato, convirtiéndolas en un grupo de enzimas con un alto potencial de aplicación en procesos biotecnológicos que hasta el momento no ha sido explotado. La presente revisión aborda aspectos generales acerca de la función y estructura de este importante grupo de enzimas.

Palabras Clave: oxigenasas, estructura-función, metaloenzimas, plasticidad funcional, centro Rieske.

\section{Structure and function of Rieske/mononuclear oxygenases}

\begin{abstract}
Rieske/mononuclear oxygenases are a group of metalloenzymes that catalyzes the oxidation of a diversity of compounds, it is noteworthy their ability to oxidize contaminant xenobiotic compounds, these enzymes also take part in the biosynthesis of some commercial valuable products. They have a broad substrate specificity that makes them a group of enzymes with a high biotechnological potential that has not been exploited to date. The present review analyzes general aspects of the structure and function of this important group of enzymes.
\end{abstract}

Key Words: oxygenases, structure-function, metalloenzymes, functional plasticity, Rieske center. 


\section{INTRODUCCIÓN}

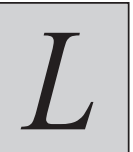

as oxigenasas son enzimas oxidoreductasas (EC 1.13 o EC 1.14) que oxidan sus sustratos introduciendo uno (monooxigenasas) o dos (dioxigenasas) átomos de oxígeno a partir del oxígeno molecular. Usualmente en su sitio activo contienen un elemento metálico el cual, a través de cambios en su estado de oxidación, activa al oxígeno para que ocurra la reacción. Fueron descritas por primera vez en un estudio sobre la fenolasa (Mason, Fowlks \& Peterson, 1955) y de la pirocatecasa (Hayaishi Katagiri \& Rothberg, 1955), enzimas que en microorganismos y animales participan en el metabolismo de ciertos compuestos como el catecol (Kojima et al., 1967) y el L-DOPA (Prabhakaran, Kirchheimer \& Harris, 1968). Desde entonces se ha reportado su participación en diversas rutas metabólicas, como la oxidación de compuestos aromáticos en bacterias (Mallick, Chakraborty \& Dutta, 2011) y la síntesis y catabolismo de diversos metabolitos en plantas (Mitchell \& Weng, 2019).

Este amplio grupo de enzimas se puede clasificar en función del cofactor que utilizan para activar el oxígeno. Están las oxigenasas dependientes de hemo, las dependientes de cobre, las dependientes de FAD-FMN y las dependientes de Fe no hémico, estas últimas se dividen en dos grupos, las enzimas que contienen dos átomos de Fe en su sitio activo y aquellas que solo tienen uno. Las que tienen dos átomos se dividen en cuatro grupos acorde a su estructura (Nordlund \& Ekland, 1995; Leahy, Batchelor \& Morcomb, 2003); mientras que las enzimas que solo tienen un átomo de Fe varían acorde al donador de electrones de este átomo, este donador puede ser un centro Rieske [2Fe-2S], una molécula de $\alpha$-cetoglutarato o una molécula de pterina (Bruijnincx, Van Koten \& Gebbink, 2008).

Las oxigenasas tipo Rieske/mononuclear, objeto de esta revisión, son enzimas que poseen en su estructura un dominio conteniendo un centro tipo Rieske [2Fe-2S] y un segundo dominio conteniendo un Fe mononuclear no hémico. Estas enzimas dependen de un sistema de transporte de electrones. Fueron descritas por primera vez como parte del sistema de degradación del benzeno en bacterias del género Pseudomonas (Axcell \& Geary, 1973 y 1975) y han sido estudiadas porque pueden oxidar diversos compuestos aromáticos xenobióticos, cuya degradación por métodos no enzimáticos es difícil.

\section{CARACTERÍSTICAS GENERALES DE LAS OXIGENASAS RIESKE/MONONUCLEAR}

El centro Rieske [2Fe-2S] fue descrito inicialmente como parte del citocromo $b c$ de una mitocondria (Rieske, MacLennan \& Coleman, 1964), en este centro metálico, un átomo de Fe está coordinado por dos residuos de cisteína y el otro átomo de $\mathrm{Fe}$ lo está por dos residuos de histidina (Figura 1A), a diferencia de los centros [2Fe-2S] clásicos en los que los cuatro residuos que coordinan a los dos átomos de Fe son cisteínas. Este centro Rieske se encuentra en el dominio que forma el amino terminal de la proteína. Los cuatro residuos de coordinación del Fe, así como la región de unión de este centro, están totalmente conservados en estas enzimas (Van Doren, Gennis, Barquera \& Crofts, 1993; Davidson, Ohnishi, Atta-Asafo-Adjei \& Daldal, 2005).

Una característica funcional importante de los centros Rieske es que su potencial de óxido-reducción varía dependiendo de sus interacciones con los aminoácidos que rodean el centro metálico y los residuos que lo coordinan (Colbert, Couture, Eltis \& Bolin, 2000). Existen centros Rieske de alto potencial redox como el del citocromo $b c$ y otros de menor potencial como el de las ferredoxinas y el de las oxigenasas Rieske/mononuclear.

El Fe mononuclear no hémico está coordinado por dos residuos de histidina y un residuo de aspártico totalmente conservados (Figura 1B). A este arreglo se le conoce como

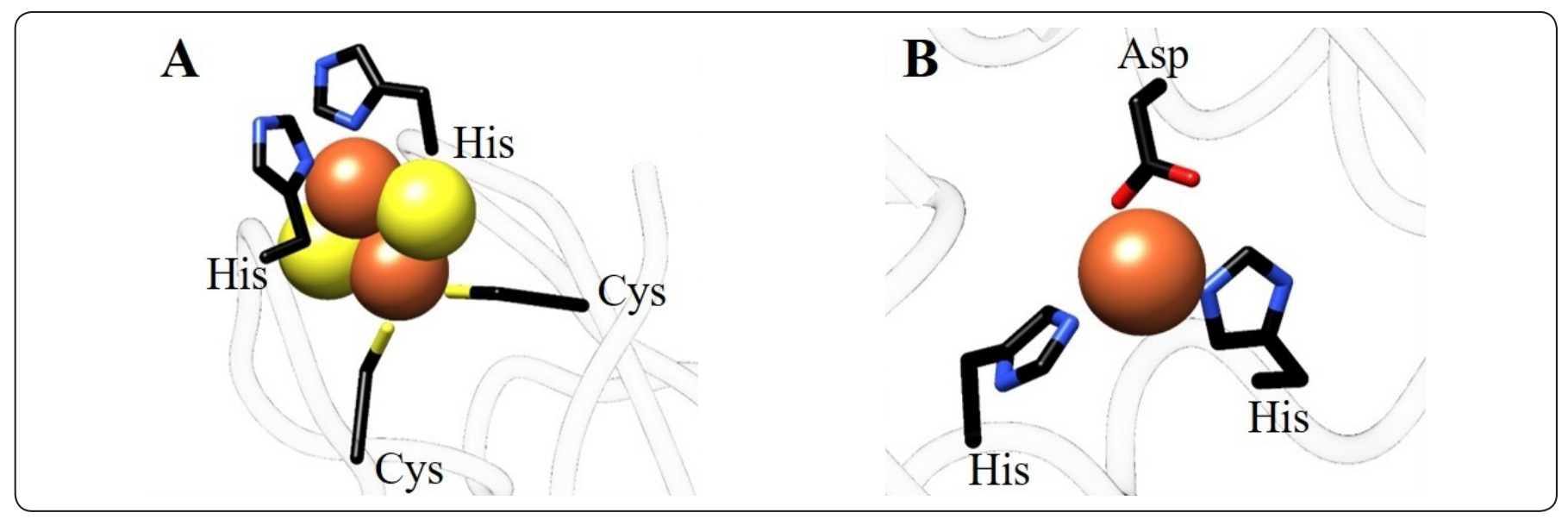

Figura 1. Coordinación de los centros metálicos en las oxigenasas Rieske/mononuclear. A) Centro Rieske [2Fe-2S]. B) Fe mononuclear no hémico. Los átomos de azufre y hierro se muestran como esferas de color amarillo y naranja respectivamente. Los residuos de aminoácidos de la proteína que coordinan a los Fe se representan como varillas, con carbonos en color negro, nitrógenos en azul y oxígenos en rojo. La figura se realizó con el software UCSF Chimera (Pettersen et al., 2004). Elaboración personal. 
la tríada 2-His-1-carboxilato, en este dominio es donde tiene lugar la activación del oxígeno y la reacción enzimática no sólo de las oxigenasas Rieske/mononuclear, sino de otros tipos de oxigenasas que tienen esta tríada (Koehntop, Emerson \& Que, 2005). El átomo de Fe se coordina de una forma octaédrica con los tres residuos previamente mencionados y tres moléculas de agua, en algunos casos la coordinación con el residuo aspártico es bidentada, uniendo solo dos moléculas de agua, pero conservando la forma octaédrica (Hegg \& Que, 1997; Que, 2000).

Las oxigenasas Rieske/mononuclear reciben los electrones del $\mathrm{NAD}(\mathrm{P}) \mathrm{H}$ a través de un sistema de transporte de electrones que en algunos casos consiste de tres componentes: una ferredoxina reductasa, cuyo grupo prostético FAD recibe los electrones del NAD(P)H y los transfiere a una ferredoxina y la oxigenasa, que recibe los electrones de la ferredoxina (Figura $2 \mathrm{~A}$ ). Mientras que en otros casos consiste en dos componentes: una reductasa dependiente de FMN o FAD y la oxigenasa (Figura 2 B). La estructura de las oxigenasas Rieske/mononuclear consiste en un homotrímero de subunidades $\alpha$ catalíticas ( $\alpha 3$ ) (Figura 3 A) o bien en un heterohexámero formado por tres subunidades $\alpha$ $\mathrm{y}$ tres subunidades $\beta$ ( $\alpha 3 \beta 3$ ) (Figura $3 \mathrm{~B}$ ). La función precisa de la subunidad $\beta$ es desconocida hasta la fecha, pero se sabe que no es esencial para la actividad catalítica, por lo que se ha propuesto que puede ayudar a la estabilidad estructural del heterohexámero o bien regular la actividad enzimática de las subunidades $\alpha$ (Hurtubise, Barriault \& Sylvestre, 1998; Kauppi et al., 1998).

Como parte del ciclo catalítico, el centro Rieske transfiere electrones al $\mathrm{Fe}$ mononuclear para la activación del oxígeno, por lo que dada la gran separación de estos centros metálicos dentro de un mismo monómero (Figura 4A), la transferencia de electrones tiene lugar desde el centro Rieske de una subunidad al Fe mononuclear de la subunidad vecina que está mucho más cercano (Figura $4 \mathrm{~B}$ ). Esta transferencia está mediada por un residuo de aspartato o glutamato de la subunidad a la que pertenece el centro $\mathrm{Fe}$ mononuclear que recibe el electrón (Parales, Parales \& Gibson, 1999). La mutación de cualquiera

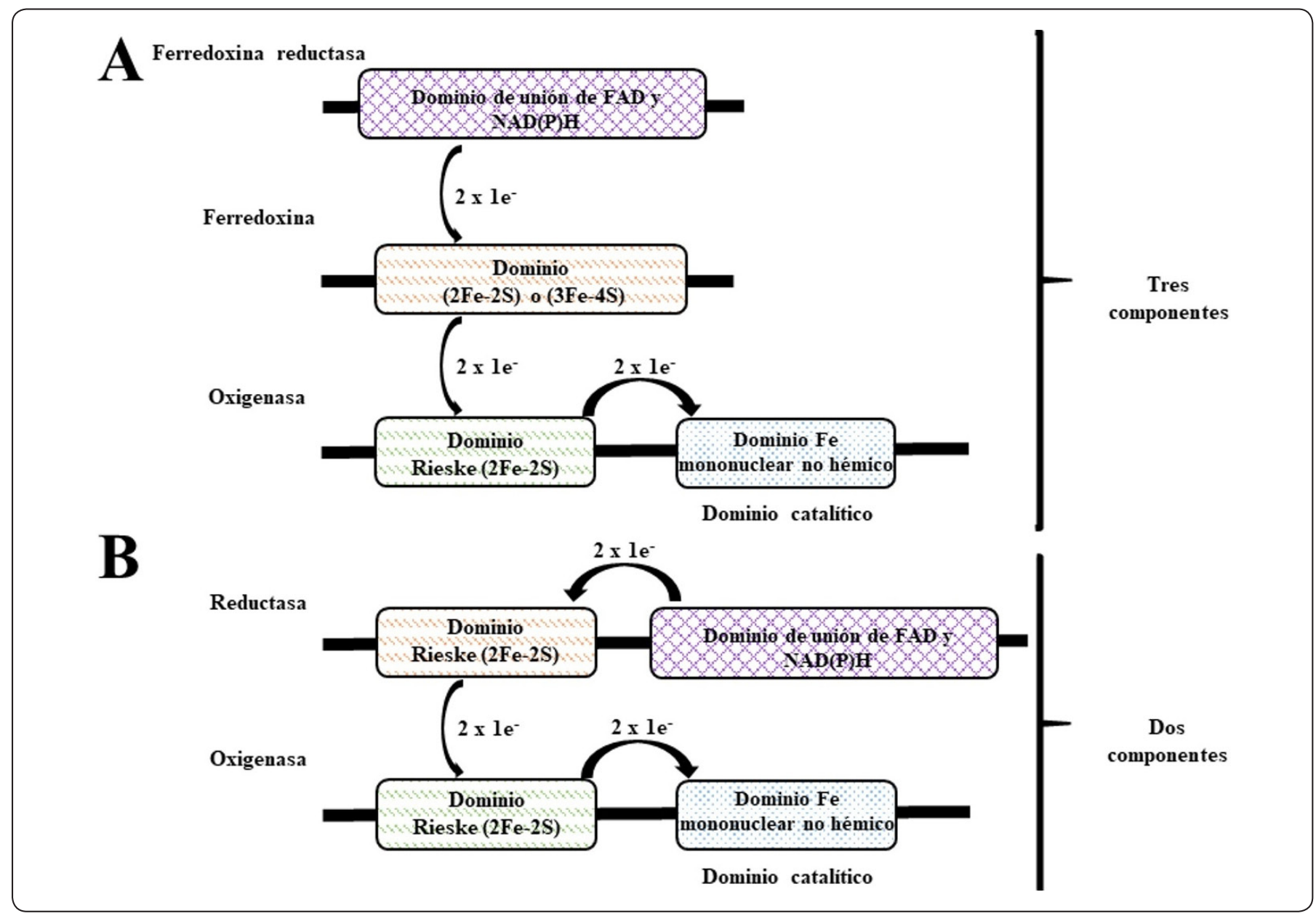

Figura 2. Representación esquemática de los tipos de oxigenasas Rieske/monuclear. (A) de tres componentes y (B) de dos componentes. Los electrones llegan a los centros Fe-S de la oxigenasa de uno en uno, pero se necesitan dos para que ocurra un ciclo catalítico. Elaboración personal. 
de los residuos que coordinan a los átomos de Fe del centro Rieske, al Fe mononuclear o del residuo ácido que participa en la transferencia de electrones entre los centros metálicos produce la pérdida de actividad de estas enzimas (Van Doren et al., 1993; Jiang, Parales, Lynch \& Gibson, 1996; Parales, Parales \& Gibson, 1999).

\section{CLASIFICACIÓN Y DISTRIBUCIÓN FILOGENÉTICA DE LAS OXIGENASAS RIESKE/MONONUCLEAR}

La base de datos de dominios conservados CDD (Conserved Domain Database, https:/www.ncbi.nlm.nih.gov/cdd), hace una clasificación por dominio de las oxigenasas Rieske/mononuclear que permite relacionar la información que se tiene acerca

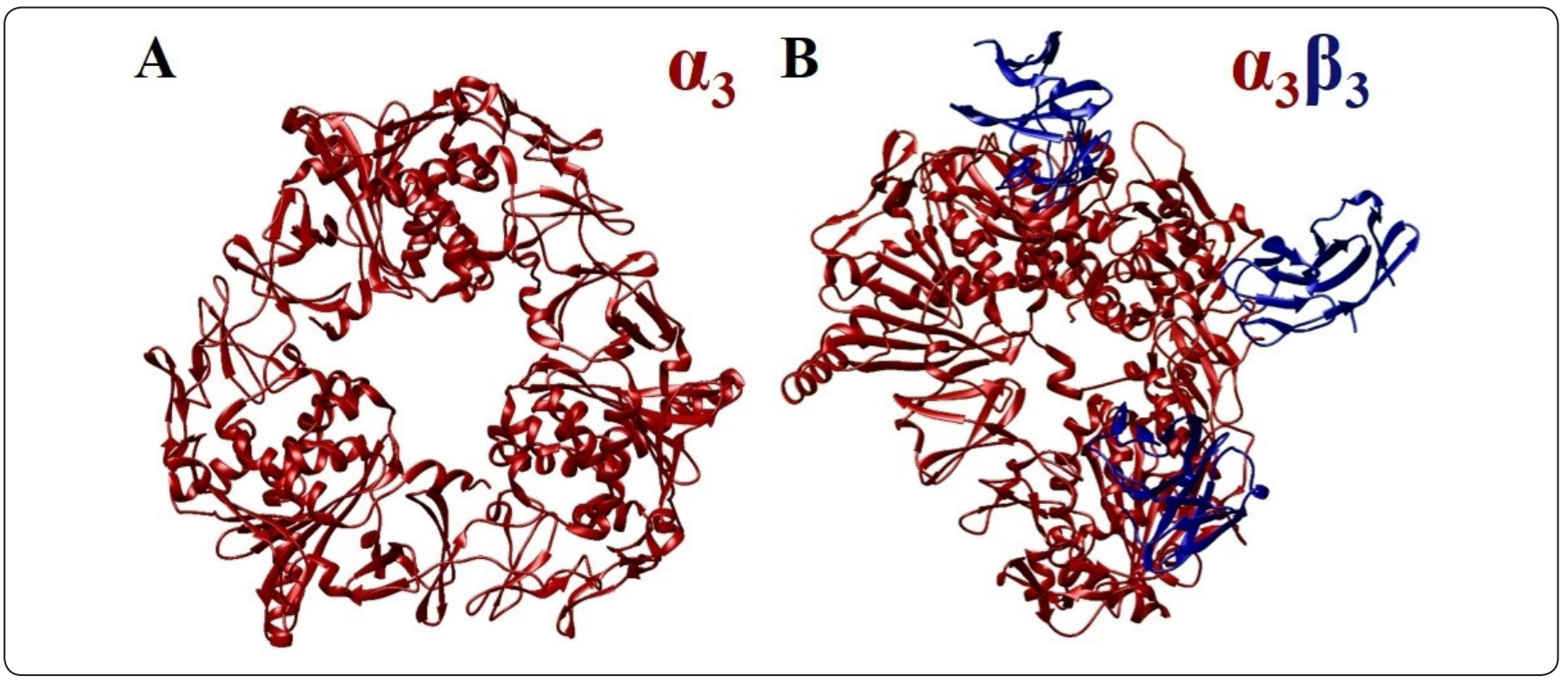

Figura 3. Representación en lazos del heterohexámero de la carbazol dioxigenasa (PDB: 2DE6). (A) Homotrímero formado por subunidades $\alpha$. (B) Heterohexámero formado por las subunidades $\alpha$ y $\beta$. La figura se realizó con el software UCSF Chimera. Elaboración personal.

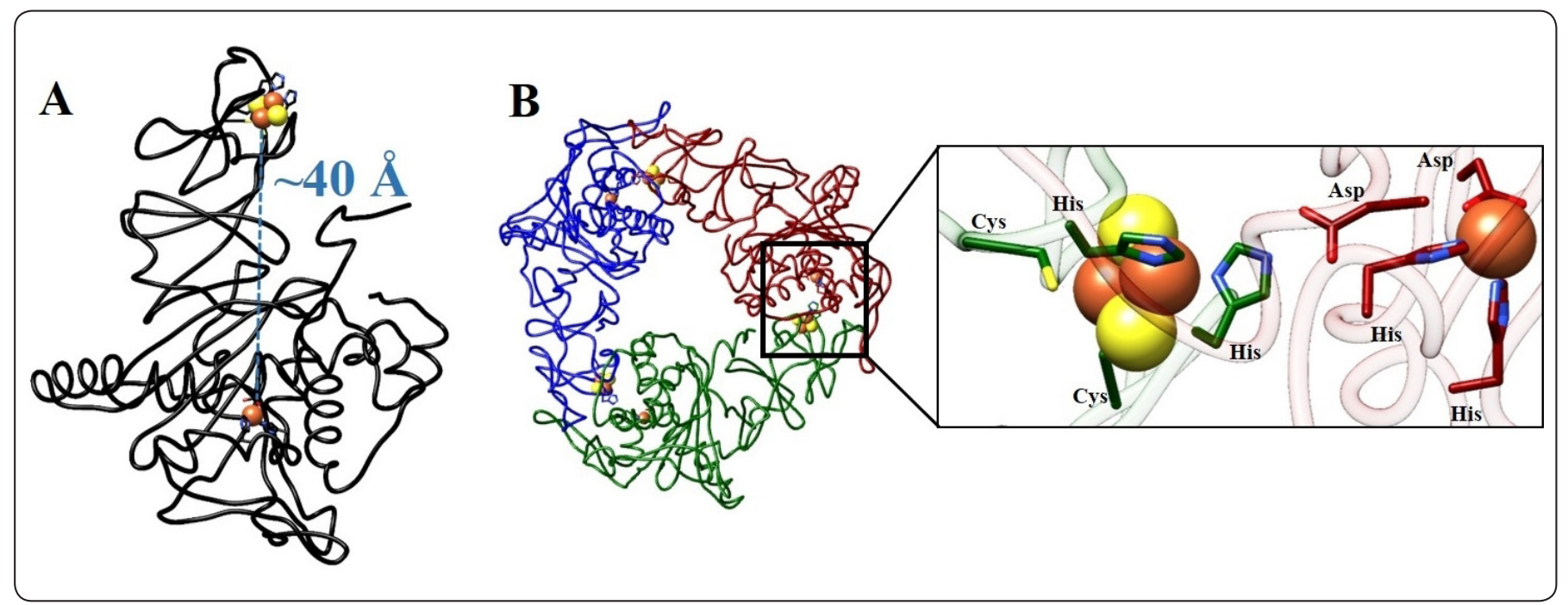

Figura 4. Transferencia de electrones entre los centros metálicos de las oxigenasas Rieske/mononuclear. A) Representación en lazos de un monómero de la oxigenasa de prolina betaína (PDB: 3VCA); con una línea punteada se indica la distancia entre sus dos centros metálicos. B) Representación en lazos del trímero de la oxigenasa de prolina betaína y acercamiento mostrando los residuos de aminoácidos que coordinan a los centros metálicos y del residuo de aspártico que asiste la transferencia de electrones desde el centro Rieske de una subunidad al Fe mononuclear no hémico de su subunidad vecina. Los residuos de aminoácidos se representan como varillas. Los carbonos se muestran en colores verde, azul o rojo cobrizo, dependiendo de la subunidad, los nitrógenos en azul y los oxígenos en rojo. Los átomos de azufre y hierro se muestran como esferas de color amarillo y naranja respectivamente. La figura se realizó con el software UCSF Chimera. Elaboración personal. 
de su estructura, función y evolución. Filogenéticamente, el dominio del centro Rieske pertenece a la familia denominada cd03469_Rieske_RO_alpha_N, que hasta la fecha comprende 16 subfamilias y el domino del Fe mononuclear no hémico pertenece a la familia denominada cd00680_RHO_alpha_C que hasta la fecha comprende 10 subfamilias (Tabla I).

Se ha propuesto otro método de clasificación de estas enzimas que toma como criterio las características estructurales del sistema de transferencia de electrones que alimenta a la oxigenasa y de esta misma. Así, se clasifican según el tipo de reductasa o de ferredoxina con el que trabajan, por ejemplo, si en la reductasa el dominio de unión del FAD/FMN se encuentra en el amino o en el carboxilo terminal o en el caso de la ferredoxina si el centro metálico es de tipo Rieske [2Fe-2S] o del tipo [3Fe-4S] y si la oxigenasa posee o no una subunidad $\beta$ (Kweon et al., 2008; Chakraborty, Ghosal, Dutta \& Dutta, 2012). El componente oxigenasa es el que juega el papel más importante en la diversificación funcional de estas enzimas, por lo que cualquier método de clasificación, debe de considerar de manera primordial a este componente.

Las oxigenasas Rieske/mononuclear están ampliamente distribuidas en bacterias (proteobacterias, actinobacterias, algunas cianobacterias y firmicutes) e incluso en algunas arqueas (Chakraborty et al., 2012) se ha descrito que estas enzimas están presentes también en bacterias termófilas, sin embargo, hasta el momento no se ha reportado la función de alguna de ellas (Chakraborty, Suzuki-Minakuchi, Okada \& Nojiri, 2017). La mayoría de las oxigenasas Rieske/mononuclear presentes en bacterias participan en rutas de degradación de compuestos aromáticos policíclicos, como el naftaleno (Ensley, Gibson, \& Laborde, 1982; Ensley \& Gibson, 1983), carbazol (Sato et al., 1997), bifenil (Haddock \& Gibson, 1995), benzoato (Wolfe et al., 2002), cumeno (Dong et al., 2005), oxoquinolina (Rosche, Tshisuaka, Fetzner \& Lingens, 1995), ftalato (Batie, Lahaie \& Ballous, 1987), benzeno (Mason, Butler, Cammack \& Shergill, 1997), tolueno (Jiang, Parales, \& Gibson, 1999), entre otros. También se han encontrado oxigenasas Rieske/mononuclear en bacterias que oxidan compuestos no aromáticos como la glicina betaína (Shao, Guo, Zhang, Yu, Zhao \& Pang, 2018), el cloruro de benzalconio (Ertekin, Konstantinidis \& Tezel, 2017), la prolina betaína (Daughtry et al., 2012) y la carnitina (Zhu et al., 2014).

En eucariontes, estas enzimas no se encuentran tan ampliamente distribuidas. En plantas, hasta la fecha se ha encontrado que participan en la síntesis del osmoprotector glicina betaína (GB)a partir de colina (Rathinasabapathi et al., 1997), en la formación de clorofila b a partir de la clorofila a y en el catabolismo de esta (Tanaka et al., 1998; Pruzinska, Tanner, Anders, Roca \& Hortensteiner, 2003). En animales, participan en la degradación del colesterol (Yoshiyama-Yanagawa et al., 2011) y en algunas levaduras en el catabolismo de colina (Linder, 2014).

\begin{tabular}{|c|c|}
\hline \multicolumn{2}{|c|}{ Familias } \\
\hline cd03469 Rieske_RO_Alpha_N & cd00680 RHO_alpha_C \\
\hline \multicolumn{2}{|c|}{ Subfamilias } \\
\hline cd03472 Rieske_RO_Alpha_BPDO_like & cd08878 RHO_alpha_C_DMO-like \\
\hline cd03479 Rieske_RO_Alpha_PhDO_like & cd08879 RHO_alpha_C_AntDO-like \\
\hline cd03480 Rieske_RO_Alpha_PaO & cd08880 RHO_alpha_C_ahdA1c-like \\
\hline cd03531 Rieske_RO_Alpha_KSH & cd08881 RHO_alpha_C_NDO-like \\
\hline cd03532 Rieske_RO_Alpha_VanA_DdmcC & cd08882 RHO_alpha_C_MupW-like \\
\hline cd03535 Rieske_RO_Alpha_NDO & cd08883 RHO_alpha_C_CMO-like \\
\hline cd03536 Rieske_RO_Alpha_DTDO & cd08884 RHO_alpha_C_GbcA-like \\
\hline cd03537 Rieske_RO_Alpha_PrnD & cd08885 RHO_alpha_C_1 \\
\hline cd03538 Rieske_RO_Alpha_AntDO & cd08886 RHO_alpha_C_2 \\
\hline cd03539 Rieske_RO_Alpha_S5H & \\
\hline cd03541 Rieske_RO_Alpha_CMO & \\
\hline cd03542 Rieske_RO_Alpha_HBDO & \\
\hline cd03545 Rieske_RO_Alpha_OHBDO_like & \\
\hline cd04337 Rieske_RO_Alpha_Cao & \\
\hline cd04338 Rieske_RO_Alpha_Tic55 & \\
\hline
\end{tabular}

Tabla I. Clasificación de las oxigenasas Rieske/mononuclear de acuerdo a la base de datos de dominios conservados. (A) Dominio Rieske, perteneciente a la familia cd03469_Rieske_RO_alpha_N (B) Dominio Fe mononuclear no hémico,perteneciente a la familia cd00680_RHO_alpha_C, En ambos casos se muestran las subfamilias que hasta el momento han sido reportadas. 
La historia evolutiva de estas enzimas está relacionada con el de proteínas multidominio, este tipo de proteínas se originan después de que ocurre algún proceso en el genoma que permite la creación de genes nuevos, entre estos procesos está la fusión génica que permite que genes con función distinta se fusionen y den origen a un gen con una nueva función (Long, Betrán, Thornton \& Wang, 2003). Aunque encontrar el mecanismo específico por el cual se originó una proteína con dos o más dominios no es sencillo, en el caso particular de las enzimas Rieske/mononuclear la fusión de dos genes que codificaban a las proteínas de función distinta pudiera ser el origen de este tipo de enzimas. El dominio Rieske pertenece a la superfamilia de proteínas que contienen hierro-azufre (ISP por sus siglas en inglés), cuyos miembros tienen un plegamiento similar y por lo tanto este dominio pudo haber surgido de una proteína ancestral cuya función estuviera relacionada con el transporte de electrones (Schmidt \& Shaw, 2001; Chakraborty \& Dutta, 2011). En el caso del dominio de unión al Fe mononuclear, su plegamiento es parecido al de las proteínas de la superfamilia denominada Betv1, cuyos miembros tienen funciones variadas. En particular se han encontrado similitudes estructurales entre la naftaleno dioxigenasa y la proteína PITP, que no tiene actividad catalítica pero media el intercambio de fosfatidilinositol y fosfatidilcolina entre diferentes membranas (Yoder et al., 2002). Tanto el dominio del Fe mononuclear no hémico de la naftaleno dioxigenasa como la PITP poseen una cavidad central, que en el caso de la PITP es donde se unen los fosfolípidos y en el de la dioxigenasa es donde se une el naftaleno (Bashton \& Chothia, 2007). También se ha propuesto que la proteína PA1206 de Pseudomonas es muy parecida estructuralmente al dominio de unión delFe mononuclear de las oxigenasas Rieske/mononuclear (Chakraborty \& Dutta, 2011). Sin embargo, ni PITP ni PA1206 unen iones metálicos y tampoco tienen conservados los residuos de unión del Fe mononuclear, totalmente conservados en las oxigenasas Rieske/mononuclear.

\section{RELACIONES ESTRUCTURA-FUNCIÓN DE LAS OXIGENASAS RIESKE/MONONUCLEAR}

Hasta el momento se han reportado estructuras cristalográficas de 13 oxigenasas Rieske/mononuclear: carbazol dioxigenasa (PDB: 2DE6, 1WW9, 3GKQ, 3GCF), dicamba monooxigenasa (PDB: 3GKE), oxoquinolina monooxigenasa (PDB: 1Z01), naftaleno dioxigenasa (PDB: 1NDO, 4HJL, 2HMO, 2B1X), bifenil dioxigenasa (PDB: 1ULI, 2GBW, 3GZX), tolueno dioxigenasa (PDB: 3EN1), cumeno dioxigenasa (PDB: $1 \mathrm{WQL}$ ), nitrobenceno dioxigenasa (PDB:2BMO), nitrotolueno dioxigenasa (PDB: 5XBP), dioxigenasa de hidrocarburos aromáticos policíclicos (PDB: 2CKF), prolina betaína monooxigenasa (PDB: 3VCA), oxigenasa de 3-cetoesteroide (PDB: 4QDC, 2ZYL) y una oxigenasa Rieske/mononuclear de función desconocida (PDB: 3N0Q). Acorde a la clasificación de la base de datos de dominios conservados del NCBI, los dominios Rieske/mononuclear de la carbazol dioxigenasa y la oxoquinolina monooxigenasa corresponden a los cd03548/ cd08878, los de la dicamba monooxigenasa a los cd03532/ cd08878, los de la naftaleno dioxigenasa, nitrotolueno dioxigenasa y nitrobenceno dioxigenasa a los cd03535/cd08881, los de la bifenil dioxigenasa, tolueno dioxigenasa, cumeno dioxigenasa y la dioxigenasa de hidrocarburos aromáticos policíclicos a los cd03472/cd08881, los de la prolina betaína monooxigenasa y la oxigenasa de función desconocida a los cd03469/cd08884 y los de la oxigenasa del 3-cetoesteroide al cd03531 sin tener aún un cd asignado para el dominio del Fe mononuclear no hémico. Es importante mencionar que las oxigenasas con los mismos dominios conservados y misma estructura tridimensional cumplen diferentes roles fisiológicos es decir, presentan una plasticidad funcional, por ejemplo la oxoquinolina monooxigenasa y la carbazol dioxigenasa tienen la misma estructura tridimensional (Figura $5 \mathrm{~A}$ ), pero están codificadas por distintos genes y cumplen roles fisiológicos distintos, la carbazol dioxigenasa tiene una amplia especificidad por el sustrato, sin embargo, no se ha estudiado si es capaz de oxidar la oxoquinolina (Nojiri et al., 1999), hasta el momento se desconoce la especificidad por el sustrato de la oxoquinolina monooxigenasa. La misma situación ocurre en el caso de la bifenil dioxigenasa y la cumeno dioxigenasa (Figura 5 B). La plasticidad funcional ha sido descrita para otras enzimas como las deshidrogenasas/reductasas de cadena media (RiverosRosas \& Julián-Sánchez, 2006), esta plasticidad funcional en las oxigenasas Rieske/mononuclear con el mismo dominio conservado se pudiera deber al grado de conservación de los residuos que conforman el sitio activo, en el caso de la carbazol dioxigenasa y naftaleno dioxigenasa (Figuras 6 A y 6 B), los residuos que se encuentran a $5 \AA$ del sitio de unión del sustrato

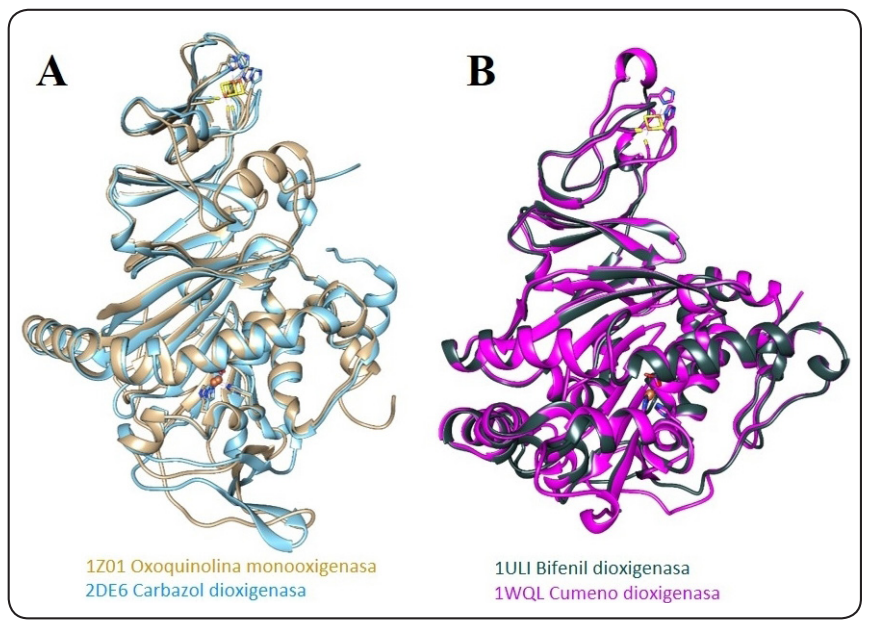

Figura 5. Diferente función, misma estructura. Comparación estructural de diferentes oxigenasas Rieske/mononuclear con los mismos dominios conservados pero diferente función. Oxoquinolina monooxigenasa y Carbazol dioxigenasa tienen los dominios conservados que pertenecen a las subfamilias cd03548/cd08878 mientras que la bifenil dioxigenasa y la cumeno dioxigenasa tienen los dominios conservados que pertenecen a las subfamilias cd03472/ cd08881. La figura se realizó con el software UCSF Chimera. Elaboración personal. 

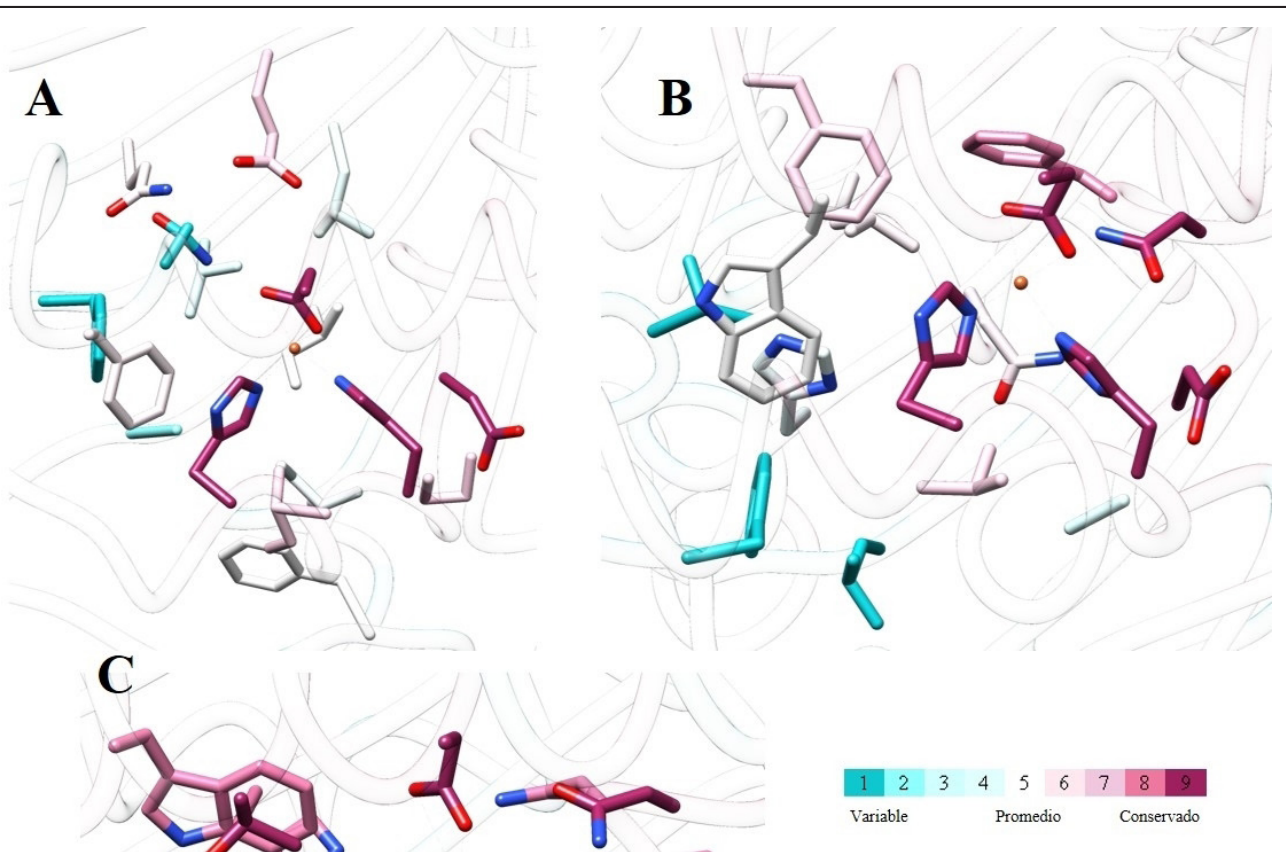

Figura 6. Conservación de aminoácidos en oxigenasas Rieske/mononuclear. Representación esquemática del sitio activo de tres oxigenasas cuya estructura se obtuvo con el sustrato unido al sitio activo, (A) PDB:2DE7 Carbazol dioxigenasa cd03548/cd08878), (B) PDB:107G Naftaleno dioxigenasa cd03535/cd0888, (C) PDB:3VCP Prolina betaína monooxigenasa cd03469/cd088841, en donde se muestran en varillas los aminoácidos a 5 Å de distancia del sitio de unión del sustrato. Los nitrógenos se muestran en azul, oxígenos en rojo y azufres en amarillo. El átomo de hierro se muestra como esfera de color naranja. La escala de conservación se muestra en la imagen como barra de colores. La figura se realizó con el software UCSF Chimera. El análisis de conservación de residuos se realizó con el programa Consurf (Ashkenazy et al., 2016). Elaboración personal.

tienen un bajo de grado de conservación, pudiendo explicar las diferentes funciones que cumplen las enzimas con los dominios cd03548/cd08878 y cd03535/cd08881. Sin embargo, en el caso de la prolina betaína monooxigenasa (cd03469/cd08884), el grado de conservación de los residuos es más elevado, se conocen dos enzimas con los mismos dominios cuya función es distinta a la de prolina betaína monooxigenasa, la glicina betaína monooxigenasa y cloruro de benzalconio monooxigenasa (Erketin et al., 2017; Shao et al., 2018).

La especificidad por el sustrato de las oxigenasas Rieske/ mononuclear es un aspecto que ha sido estudiado en algunas de ellas, por ejemplo, la bifenil dioxigenasa de Pseudomonas sp. LB400 es capaz de oxidar mayor variedad de bifenilos policlorados que la de Pseudomonas pseudoalcaligenes KF707, (Gibson, Cruden, Haddock, Zylstra \& Brand, 1993), las enzimas son diferentes entre ellas en sólo 20 residuos, la mutación de un solo residuo en el sitio activo de la enzima de $P$. pseudoalcaligenes KF707 permite cambiar de manera considerable la especificidad por el sustrato de esta (Suenaga, Goto \& Furukawa, 2006). La carbazol dioxigenasa tiene la capacidad de oxidar compuestos que son estructuralmente parecidos al carbazol pero en algunos casos como el fluoranteno no lo son y también los oxida, esta enzima puede incluso oxidar al bifenilo (Nojiri et al., 1999), esta última oxidación también la lleva a cabo la naftaleno dioxigenasa, dependiendo del sustrato, esta enzima puede llevar a cabo reacciones de monooxigenación, desaturación, sulfoxidación y desalquilación confiriéndole una capacidad de oxidación de compuestos mucho más amplia (Resnick, Lee \& Gibson, 1996). La especificidad por el sustrato de estas enzimas está determinada principalmente por los residuos que forman el sitio de unión de este, la forma en que potenciales sustratos se unan a este y el cómo la unión de estos afecte el ambiente 
del Fe mononuclear. Una afectación en este ambiente puede causar cambios en la carga de este átomo y modificar la manera en que el sustrato o potencial sustrato se una al sitio, causando también cambios en la estabilidad y/o forma de unión de los intermediarios de la reacción enzimática (Wang, Li \& Liu, 2017), otro factor importante es el tamaño del sitio, por ejemplo, la oxigenasa de compuestos aromáticos policiclicos de Sphingobium sp. PNB tiene un sitio de unión al sustrato bastante más grande que el de otras oxigenasas, lo que le permite oxidar compuestos de diversos tamaños (Khara, Roy, Chakraborty, Dutta \& Dutta, 2018).

\section{RETOS EN EL USO Y APLICACIÓN DE LAS OXIGENASAS RIESKE/MONONUCLEAR}

Es importante tener en cuenta que la intención de usar en procesos de tipo industrial a cualquier biocatalizador como lo son las enzimas conlleva ciertos retos. Respecto a las oxigenasas, Van Beilen, Duetz, Schmid \& Witholt (2003), mencionan que se deben de considerar aspectos como la capacidad de obtener de manera heteróloga a la enzima, la cantidad de enzima recombinante que se puede obtener, la eficiencia catalítica, la eficiencia de unión del sustrato y producto de la reacción enzimática, la transferencia de oxígeno en el medio y su disponibilidad para la enzima, la posibilidad de que los productos de la reacción enzimática sean oxidados de nuevo, la disponibilidad de cofactores y la solubilidad de los sustratos. En el caso de las oxigenasas Rieske/mononuclear, dado que la mayoría de estas enzimas son de origen procarionte los problemas respecto a la obtención de manera heteróloga de estas enzimas y la cantidad de enzima recombinante pudieran ser aspectos fáciles de atender, de hecho, enzimas como la ftalato dioxigenasa de Burkholderia cepacia y la carbazol dioxigenasa de Pseudomonas stutzeri han logrado ser obtenidas de forma recombinante a niveles de gramos por litro en cultivos líquidos (Jaganaman, Pinto, Tarasev \& Ballou, 2007; Larentis, Sampaio, Martins, Rodrigues \& Alves, 2011). A través de la ingeniería de proteínas se ha logrado que algunas oxigenasas Rieske/mononuclear utilicen un mayor número de moléculas y se ha logrado también aumentar la eficiencia catalítica (Özgen \& Schmidt, 2019). Un factor que pudiera ser importante a considerar es la disponibilidad de cofactores, dada la naturaleza del sistema de transferencia de electrones de estas enzimas, es necesario contar con cofactores como el NAD(P)H y el FMN/FAD, estas moléculas son costosas y por lo tanto supondrían una limitante en el uso y aplicación de las oxigenasas Rieske/mononuclear, una alternativa sería el uso de cofactores sintéticos menos costosos que mimetizan la función de los cofactores biológicos (Zachos, Nowak \& Sieber, 2019), en ambos casos es importante considerar que es necesario reducir estas moléculas, existen diversos métodos probados mediante los cuales se puede lograr ésto (Wu et al., 2013), un escenario ideal sería aquel en donde los centros metálicos de la oxigenasa pudieran ser reducidos directamente sin la necesidad de los otros componentes del sistema de transferencia de electrones, en este sentido, compuestos químicos como la ditionita de sodio pueden lograr este fin, sin embargo, el costo de su uso de nueva cuenta impone una limitante.

\section{Potenciales APliCACIONES BIOTECNOLÓGICAS DE LAS OXIGENASAS RIESKE/MONONUCLEAT}

Las oxigenasas Rieske/mononuclear de bacterias han sido utilizadas para sintetizar dos compuestos con alto valor comercial: el colorante índigo, muy usado en la industria textil, y el fármaco indinavir, un inhibidor de una proteasa del VIH con aplicaciones terapéuticas. La síntesis química del colorante índigo, además de ser laboriosa, utiliza y produce compuestos químicos altamente contaminantes (Bialy, 1997). La biosíntesis de este compuesto se basa en la oxidación del indol (un intermediario de la ruta de biosíntesis del triptófano, Ensley et al., 1983) y no solo produce menos compuestos contaminantes, sino que económicamente es más conveniente que la síntesis química. Un importante intermediario en la síntesis del indinavir se puede obtener mediante la enzima tolueno dioxigenasa (Wackett, Kwart \& Gibson, 1988).

Probablemente el área en la que la participación de las oxigenasas Rieske/mononuclear podría destacar más es en la de biorremediación, en particular las oxigenasas de bacterias. A pesar de la capacidad de estas enzimas de oxidar compuestos policíclicos aromáticos, hay poca información acerca de su aplicación o uso directo para remediar áreas contaminadas con dichos compuestos. Sin embargo, en lodos activados de plantas de tratamiento de aguas se ha encontrado que la comunidad de microorganismos presente posee una diversidad de oxigenasas importante que permite a esta comunidad degradar compuestos aromáticos (Jadeja, More, Purohit \& Kapley, 2014), incluyendo bacterias previamente desconocidas con capacidad para degradar compuestos aromáticos (Li et al., 2017). Se han estudiado comunidades de microorganismos en áreas contaminadas con diferentes compuestos, encontrándose que hay una importante diversidad de microorganismos cultivables y no cultivables que forman parte de dichas comunidades y que poseen una gran cantidad de genes de oxigenasas Rieske/mononuclear, muchos de los cuales codifican para oxigenasas de las que no se han descrito homólogos cercanos (Ni Chadhain, Norman, Pesce, Kukor \& Zylstra, 2006). Estas comunidades pueden ser estimuladas añadiendo algún compuesto en particular como el naftaleno, lo que permite la selección de microorganismos con una mayor capacidad para degradar este compuesto (Ni Chadhain et al., 2006). Además, estas comunidades son muy estables durante períodos prolongados de tiempo, lo cual es ideal para la biorremediación de áreas altamente contaminadas (Yagi \& Madsen, 2009) y pueden tolerar cambios en el ambiente causantes de estrés abiótico, tales como, el aumento en la salinidad de los suelos (Li et al., 2012). Dada la gran cantidad de secuencias de oxigenasas Rieske/mononuclear recientemente obtenidas existe la oportunidad de encontrar nuevas funciones, 
enzimas con una mayor eficiencia catalítica o una especificidad por el sustrato más amplia (Martin, Malagnoux, Violet, Jakoncic \& Jouanneau, 2012).

Otra área de desarrollo potencial de aplicación de las oxigenasas Rieske/mononuclear tiene como base la enzima que participa en el catabolismo de la cafeína en Pseudomonas putida CBB5, una bacteria que puede incluso utilizar la cafeína como única fuente de carbono y nitrógeno (Summers et al., 2012). A pesar de que la capacidad de ciertos microorganismos de metabolizar cafeína ha sido estudiada por bastante tiempo, la participación de las oxigenasas Rieske/mononuclear no había sido establecida hasta hace poco tiempo y ello plantea la posibilidad de utilizar estas enzimas o incluso los microorganismos que poseen los genes para las mismas, para llevar a cabo procesos como el bio-descafeínado, biorremediación de suelos contaminados con cafeína o síntesis de metabolitos con valor comercial que forman parte del catabolismo de la cafeína (Summers, Mohanty, Gopishetty, \& Subramanian, 2015).

En conclusión, las oxigenasas Rieske/mononuclear son enzimas con una amplia diversidad funcional y dado que su aparentemente más extendido rol fisiológico es la oxidación de compuestos aromáticos policíclicos tóxicos o de interés industrial, constituyen una potencial herramienta biotecnológica que hasta el momento no se ha explotado. Otra área de potencial desarrollo es el uso de estas enzimas para la síntesis de compuestos importantes para diferentes industrias o en el área de la salud. El número de genes y secuencias de proteínas de enzimas Rieske/mononuclear son mucho más grandes que el número de enzimas bioquímica o estructuralmente caracterizadas o con función comprobada hasta el momento, lo cual es una gran ventana de oportunidad para seguir estudiando este importante grupo de enzimas.

\section{Agradecimientos}

El autor agradece al CONACYT por la beca de doctorado otorgada. Se extiende un agradecimiento a la Doctora Rosario A. Muñoz Clares y al doctor Héctor Riveros Rosas por la revisión crítica, los comentarios y sugerencias realizados para mejorar el escrito.

\section{REFERENCIAS}

Ashkenazy, H., Abadi, S., Martz, E., Chay, O., Mayrose, I., Pupko, T. \& Ben-Tal, N. (2016). ConSurf2016: an improved methodology to estimate and visualize evolutionary conservation in macromolecules. Nucleic Acids Research, 44(W1), W344-W350. https://doi.org/10.1093/nar/ gkw408.

Axcell, B. C. \& Geary, P. J. (1973). The metabolism of benzene by bacteria. Purification and some properties of the enzyme cis-1,2-dihydroxycyclohexa-3,5-diene (nicotinamide adenine dinucleotide) oxidoreductase (cis-benzene glycol dehydrogenase). Biochemical Journal, 136(4), 927-934.
Axcell, B. C. \& Geary, P. J. (1975). Purification and some properties of a soluble benzene-oxidizing system from a strain of Pseudomonas. Biochemical Journal, 146(1), 173-183. https://doi.org/DOI 10.1111/j.14321033.1997.00833.x.

Bashton, M. \& Chothia, C. (2007). The generation of new protein functions by the combination of domains. Structure, 15(1), 85-99. https://doi.org/10.1016/j.str.2006.11.009.

Batie, C. J., Lahaie, E. \& Ballous, P. (1987). Purification and characterization of phthalate oxygenase and phtalate oxygenase reductase from Pseudomonas cepacia. Journal of Biological Chemistry, 262(4), 1510-1518.

Bialy, H. (1997). Biotechnology, bioremediation, and blue genes. Nature Biotechnology, 15(2), 110. https://doi.org/10.1038/ nbt0297-110.

Bruijnincx, P. C. A., Van Koten, G. \& Gebbink, R. J. M. K. (2008). Mononuclear non-heme iron enzymes with the 2-His-1-carboxylate facial triad: Recent developments in enzymology and modeling studies. Chemical Society Reviews, 37(12), 2716-2744.

Chakraborty, J. \& Dutta, T. K. (2011). From lipid transport to oxygenation of aromatic compounds: evolution within the Bet v1-like superfamily. Journal of Biomolecular Structure and Dynamics, 29(1), 67-78. https://doi.org/10.1080/073 91102.2011.10507375.

Chakraborty, J., Ghosal, D., Dutta, A. \& Dutta, T. K. (2012). An insight into the origin and functional evolution of bacterial aromatic ring-hydroxylating oxygenases. Journal of Biomolecular Structure and Dynamics, 30(4), 419-436. https://doi.org/10.1080/07391102.2012.682208.

Chakraborty, J., Suzuki-Minakuchi, C., Okada, K. \& Nojiri, H. (2017). Thermophilic bacteria are potential sources of novel Rieske non-heme iron oxygenases. AMB Express, 7(1). https://doi.org/10.1186/s13568-016-0318-5.

Colbert, C. L., Couture, M. M. J., Eltis, L. D. \& Bolin, J. T. (2000). Acluster exposed: structure of the Rieske ferredoxin from biphenyl dioxygenase and the redox properties of Rieske Fe-S proteins. Structure, 8(12), 1267-1278. https:// doi.org/10.1016/S0969-2126(00)00536-0.

Daughtry, K. D., Xiao, Y., Stoner-Ma, D., Cho, E., Orville, A. M., Liu, P. \& Allen, K. N. (2012). Quaternary ammonium oxidative demethylation: X-ray crystallographic, resonance raman, and uv-visible spectroscopic analysis of a Riesketype demethylase. Journal of the American Chemical Society, 134(5), 2823-2834. https://doi.org/10.1021/ ja2111898.

Davidson, E., Ohnishi, T., Atta-Asafo-Adjei, E. \& Daldal, F. (2005). Potential ligands to the [2Fe-2S] Rieske cluster of the cytochrome $b c 1$ complex of Rhodobacter capsulatus probed by site-directed mutagenesis. Biochemistry, 31(13), 3342-3351. https://doi.org/10.1021/bi00128a006.

Dong, X., Fushinobu, S., Fukuda, E., Terada, T., Nakamura, S., Shimizu, K., Nojiri, H., Omori, T., Shoun, H. \& Wakagi, T. (2005). Crystal structure of the terminal oxygenase 
component of cumene dioxygenase from Pseudomonas fluorescens IP01. Journal of Bacteriology, 187(7), 24832490. https://doi.org/10.1128/JB.187.7.2483-2490.2005.

Ensley, B. D. \& Gibson, D. T. (1983). Naphthalene dioxygenase: purification and properties of a terminal oxygenase component. Journal of Bacteriology, 155(2), 505-511.

Ensley, B. D., Gibson, D. T. \& Laborde, A. L. (1982). Oxidation of naphthalene by a multicomponent enzyme system from Pseudomonas sp. strain NCIB 9816. Journal of Bacteriology, 149(3), 948-954.

Ensley, B. D., Ratzkin, B. J., Osslund, T. D., Simon, M. J., Wackett, L. P. \& Gibson, D. T. (1983). Expression of naphthalene oxidation genes in Escherichia coli results in the biosynthesis of indigo. Science, 222(4620), 167-169. https://doi.org/10.1126/science.6353574.

Ertekin, E., Konstantinidis, K. T.,\& Tezel, U. (2017). A Rieske-type oxygenase of Pseudomonas sp. BIOMIG1 converts benzalkonium chlorides to benzyldimethyl amine. Environmental Science and Technology, 51(1), 175-181. https://doi.org/10.1021/acs.est.6b03705.

Gibson, D. T., Cruden, D. L., Haddock, J. D., Zylstra, G. J. \& Brand, J. M. (1993). Oxidation of polychlorinated biphenyls by Pseudomonas sp. strain LB400 and Pseudomonas pseudoalcaligenes KF707. Journal of Bacteriology, 175(14), 4561-4564. https://doi.org/10.1128/ jb.175.14.4561-4564.1993.

Haddock, J. D. \& Gibson, D. T. (1995). Purification and characterization of the oxygenase component of biphenyl 2,3-dioxygenase from Pseudomonas sp. strain LB400 [published erratum appears in J. Bacteriol. 1996 Apr;178(7):258]. Journal of Bacteriology, 177(20), 5834-5839.

Hayaishi, O., Katagiri, M. \& Rothberg, S. (1955). Mechanism of the pyrocatechase reaction. Journal of the American Chemical Society, 77(20), 5450-5451. https://doi. org/10.1021/ja01625a095.

Hegg, E. L. \& Jr, L. Q. (1997). The 2-His-1-carboxylate facial triad - an emerging structural motif in mononuclear nonheme iron(II) enzymes. European Journal of Biochemistry, 250(3), 625-629.https://doi.org/10.1111/j.1432-1033.1997. t01-1-00625.x.

Hurtubise, Y., Barriault, D. \& Sylvestre, M. (1998). Involvement of the terminal oxygenase $\beta$ subunit in the biphenyl dioxygenase reactivity pattern toward chlorobiphenyls. Journal of Bacteriology, 180(22), 5828-5835.

Jadeja, N. B., More, R. P., Purohit, H. J. \& Kapley, A. (2014). Metagenomic analysis of oxygenases from activated sludge. Bioresource Technology, 165, 250-256. https:// doi.org/10.1016/j.biortech.2014.02.045.

Jaganaman, S., Pinto, A., Tarasev, M. \& Ballou, D. P. (2007). High levels of expression of the iron-sulfur proteins phthalate dioxygenase and phthalate dioxygenase reductase in Escherichia coli. Protein Expression and Purification, 52(2), 273-279. https://doi.org/10.1016/j.pep.2006.09.004.
Jiang, H., Parales, R. E. \& Gibson, D. T. (1999). The $\alpha$ subunit of toluene dioxygenase from Pseudomonas putida F1 can accept electrons from reduced ferredoxin (TOL) but is catalytically inactive in the absence of the $\beta$ subunit. Applied and Environmental Microbiology, 65(1), 315-318.

Jiang, H., Parales, R. E., Lynch, N. A. \& Gibson, D. T. (1996). Site-directed mutagenesis of conserved amino acids in the alpha subunit of toluene dioxygenase: potential mononuclear non-heme iron coordination sites. Journal of Bacteriology, 178(11),3133-3139.https://doi.org/10.1128/ jb.178.11.3133-3139.1996.

Kauppi, B., Lee, K., Carredano, E., Parales, R. E., Gibson, D. T., Eklund, H. \& Ramaswamy, S. (1998). Structure of an aromatic-ring-hydroxylating dioxygenase naphthalene 1,2-dioxygenase. Structure, 6(5), 571-586. https://doi. org/10.1016/S0969-2126(98)00059-8.

Khara, P., Roy, M., Chakraborty, J., Dutta, A. \& Dutta, T. K. (2018). Characterization of a topologically unique oxygenase from Sphingobium sp. PNB capable of catalyzing a broad spectrum of aromatics. Enzyme and Microbial Technology, 111, 74-80. https://doi.org/10.1016/j. enzmictec.2017.10.006.

Koehntop, K. D., Emerson, J. P. \& Que, L. (2005). The 2-His1-carboxylate facial triad: a versatile platform for dioxygen activation by mononuclear non-heme iron (II) enzymes. Journal of Biological Inorganic Chemistry, 10(2), 87-93. https://doi.org/10.1007/s00775-005-0624-x.

Kojima, Y., Fujisawa, H., Nakazawa, A., Nakazawa, T., Kanetsuna, F., Taniuchi, H., Nozaki, M. \& Hayaishi, O. (1967). Studies on Pyrocatechase. The Journal of Biological Chemistry, 242(14), 3270-3278.

Kweon, O., Kim, S. J., Baek, S., Chae, J. C., Adjei, M. D., Baek, D. H., Kim, Y. C. \& Cerniglia, C. E. (2008). A new classification system for bacterial Rieske non-heme iron aromatic ring-hydroxylating oxygenases. $B M C$ Biochemistry, 9(1), 1-20. https://doi.org/10.1186/14712091-9-11.

Larentis, A. L., Sampaio, H. D. C. C., Martins, O. B., Rodrigues, M. I. \& Alves, T. L. M. (2011). Influence of induction conditions on the expression of carbazole dioxygenase components (CarAa, CarAc, and CarAd) from Pseudomonas stutzeri in recombinant Escherichia coli using experimental design. Journal of Industrial Microbiology and Biotechnology, 38(8), 1045-1054. https://doi.org/10.1007/s10295-010-0879-2.

Leahy, J. G., Batchelor, P. J. \& Morcomb, S. M. (2003). Evolution of the soluble diiron monooxygenases. FEMSMicrobiology Reviews, 27(4), 449-479. https://doi.org/10.1016/S01686445(03)00023-8.

Li, H., Zhang, Q., Wang, X. L., Ma, X. Y., Lin, K. F., Liu, Y. D., Gu, J. D., Lu, S. G., Shi, L., Lu, Q. \& Shen, T. T. (2012). Biodegradation of benzene homologues in contaminated sediment of the east China sea. Bioresource Technology, 124, 129-136. https://doi.org/10.1016/j.biortech.2012.08.033. 
Li, J., Zhang, D., Song, M., Jiang, L., Wang, Y., Luo, C. \& Zhang, G. (2017). Novel bacteria capable of degrading phenanthrene in activated sludge revealed by stableisotope probing coupled with high-throughput sequencing. Biodegradation, 28(5-6), 423-436. https://doi.org/10.1007/ s10532-017-9806-9.

Linder, T. (2014). CMO1 encodes a putative choline monooxygenase and is required for the utilization of choline as the sole nitrogen source in the yeast Scheffersomyces stipitis (syn. Pichia stipitis). Microbiology (United Kingdom), 160(PART 5), 929-940. https://doi.org/10.1099/ mic.0.073932-0.

Long, M., Betrán, E., Thornton, K. \& Wang, W. (2003). The origin of new genes: glimpses from the young and old. Nature Reviews Genetics, 4(11), 865-875. https://doi. org/10.1038/nrg1204.

Mallick, S., Chakraborty, J. \& Dutta, T. K. (2011). Role of oxygenases in guiding diverse metabolic pathways in the bacterial degradation of low-molecular-weight polycyclic aromatic hydrocarbons: a review. Critical Reviews in Microbiology, 37(1), 64-90. https://doi.org/10.3109/104 0841X.2010.512268.

Martin, F., Malagnoux, L., Violet, F., Jakoncic, J. \& Jouanneau, Y. (2012). Diversity and catalytic potential of PAH-specific ring-hydroxylating dioxygenases from a hydrocarbon-contaminated soil. Applied Microbiologyand Biotechnology,97(11),5125-5135.https://doi.org/10.1007/ s00253-012-4335-2.

Mason, H. S., Fowlks, W. L. \& Peterson, E. (1955). Oxygen transfer and electron transport by the phenolase complex. Journal of the American Chemical Society, 77(10), 2914 2915. https://doi.org/10.1021/ja01615a088.

Mason, J. R., Butler, C. S., Cammack, R. \& Shergill, J. K. (1997). Structural studies on the catalytic component of benzene dioxygenase from Pseudomonas putida. Biochemical Society Transactions, 25(1), 90-95. https:// doi.org/10.1042/bst0250090.

Mitchell, A. J. \& Weng, J.-K. (2019). Unleashing the synthetic power of plant oxygenases: from mechanism to application. Plant Physiology, 179(March), pp.01223.2018. https://doi. org/10.1104/pp.18.01223.

Ni Chadhain, S. M., Norman, S. R., Pesce, K. V., Kukor, J. J. \& Zylstra, G. J. (2006). Microbial dioxygenase gene population shifts during polycyclic aromatic hydrocarbon biodegradation. Applied and Environmental Microbiology, 72(6), 4078-4087. https://doi.org/10.1128/aem.02969-05.

Nojiri, H., Nam, J. W., Kosaka, M., Morii, K. I., Takemura, T., Furihata, K., Yamane, H. \& Omori, T. (1999). Diverse oxygenations catalyzed by carbazole 1,9a-dioxygenase from Pseudomonas sp. strain CA10. Journal of Bacteriology, 181(10), 3105-3113.

Nordlund, P. \& Eklund, H. (1995). Di-iron-carboxylate proteins. Current Opinion in Structural Biology, 5(6), 758-766. https://doi.org/10.1016/0959-440X(95)80008-5.
Özgen, F. F. \& Schmidt, S. (2019). Rieske Non-Heme Iron Dioxygenases: Applications and Future Perspectives. In Q. Husain \& M.F. Ullah (Eds.), Biocatalysis: Enzymatic Basics and Applications (pp. 57-82). https://doi.org/10.1007/9783-030-25023-2 4.

Parales, R. E., Parales, J. V. \& Gibson, D. T. (1999). Aspartate 205 in the catalytic domain of naphthalene dioxygenase is essential for activity. Journal of Bacteriology, 181(6), 1831-1837.

Pettersen, E. F., Goddard, T. D., Huang, C. C., Couch, G. S., Greenblatt, D. M., Meng, E. C.\& Ferrin, T.E. (2004). UCSF Chimera - A visualization system for exploratory research and analysis. Journal of Computational Chemistry, 25(13), 1605-1612. https://doi.org/10.1002/jcc.20084.

Prabhakaran, K., Kirchheimer, W. F. \& Harris, E. B. (1968). Oxidation of phenolic compounds by Mycobacterium leprae and inhibition of phenolase by substrate analogues and copper chelators. Journal of Bacteriology, 95(6), 2051-2053.

Pruzinska, A., Tanner, G., Anders, I., Roca, M. \& Hortensteiner, S. (2003). Chlorophyll breakdown: pheophorbide a oxygenase is a Rieske-type iron-sulfur protein, encoded by the accelerated cell death 1 gene. Proceedings of the National Academy of Sciences, 100(25), 15259-15264. https://doi.org/10.1073/ pnas. 2036571100 .

Que, L. (2000). One motif - many different reactions. Nature Structural Biology, 7(3), 182-184. https://doi. org/10.1038/73270.

Rathinasabapathi, B., Burnet, M., Russell, B. L., Gage, D. A., Liao, P.-C., Nye, G. J., Scott, P., Golbeck, J. H. \& Hanson, A. D. (1997). Choline monooxygenase, an unusual ironsulfur enzyme catalyzing the first step of glycine betaine synthesis in plants: prosthetic group characterization and cDNA cloning. Proceedings of the National Academy of Sciences, 94(7), 3454-3458. https://doi.org/10.1073/ pnas.94.7.3454.

Resnick, S., Lee, K. \& Gibson, D. (1996). Diverse reactions catalyzed by naphthalene dioxygenase from Pseudomonas sp strain NCIB 9816. Journal of Industrial Microbiology \& Biotechnology, 17(5-6), 438-457.https://doi.org/10.1007/ bf01574775.

Rieske, J. S., MacLennan, D. H. \& Coleman, R. (1964). Isolation and properties of an iron-protein from the (reduced coenzyme Q)-cytochrome $\mathrm{C}$ reductase complex of the respiratory chain. Biochemical and Biophysical Research Communications, 15(4), 338-344. https://doi. org/10.1016/0006-291X(64)90171-8.

Riveros-Rosas, H. \& Julián-Sánchez, A. (2006). Functional plasticity of medium-chain dehydrogenases / reductases. In H. Weiner, B. Plapp, R. Londahl, \& E. Maser (Eds.), Enzymology and Molecular Biology of Carbonyl Metabolism 12 (pp. 419-433). West Lafayette, Indiana: Purdue University. 
Rosche, B., Tshisuaka, B., Fetzner, S. \& Lingens, F. (1995). 2-Oxo-1,2-dihydroquinoline 8-Monooxygenase, a twocomponent enzyme system from Pseudomonas putida 86. The Journal of Biological Chemistry, 270(30), 17836-17842. https://doi.org/10.1074/jbc.270.30.17836.

Sato, S., Nam, J., Kasuga, K., Nojiri, H., Yamane, H., \& Omori, T. (1997). Identification and characterization of genes encoding carbazole 1,9a-dioxygenase in Pseudomonas sp. strain CA10. Journal of Bacteriology, 179(15), 4850-4858.

Schmidt, C.L.\& Shaw, L. (2001). Acomprehensive phylogenetic analysis of Rieske and Rieske-type iron-sulfur proteins. Journal of Bioenergetics and Biomembranes, 33(1), 9-26. https://doi.org/10.1023/A:1005616505962.

Shao, Y.-H., Li-Zhong Guo., Yu-Qing Zhang, Hao Yu, Bai-Suo Zhao \& Hai-Qiang Pang, W.-D. L. (2018). Glycine betaine monooxygenase, an unusual Rieske-type oxygenase system, catalyzes the oxidative N-Demethylation of glycine betaine in Chromohalobacter salexigens DSM 3043. Applied and Environmental Microbiology, 84(13), 1-18.

Suenaga, H., Goto, M. \& Furukawa, K. (2006). Active-site engineering of biphenyl dioxygenase: effect of substituted amino acids on substrate specificity and regiospecificity. Applied Microbiology and Biotechnology, 71(2), 168-176. https://doi.org/10.1007/s00253-005-0135-2.

Summers, R. M., Louie, T. M., Yu, C. L., Gakhar, L., Louie, K. C. \& Subramanian, M. (2012). Novel, highly specific $\mathrm{N}$-Demethylases enable bacteria to live on caffeine and related purine alkaloids. Journal of Bacteriology, 194(8), 2041-2049. https://doi.org/10.1128/jb.06637-11.

Summers, R. M., Mohanty, S. K., Gopishetty, S. \& Subramanian, M. (2015). Genetic characterization of caffeine degradation by bacteria and its potential applications. Microbial Biotechnology, 8(3), 369-378. https://doi.org/10.1111/1751-7915.12262.

Tanaka, A., Ito, H., Tanaka, R., Tanaka, N. K., Yoshida, K. \& Okada, K. (1998). Chlorophyll a oxygenase (CAO) is involved in chlorophyll b formation from chlorophyll a. Proceedings of the National Academy of Sciences, 95(21), 12719-12723. https://doi.org/10.1073/pnas.95.21.12719.

Van Beilen, J. B., Duetz, W.A., Schmid, A. \& Witholt, B. (2003). Practical issues in the application of oxygenases. Trends in Biotechnology, 21(4), 170-177. https://doi.org/10.1016/ S0167-7799(03)00032-5.

Van Doren, S. R., Gennis, R. B., Barquera, B. \& Crofts, A. R. (1993). Site-directed mutations of conserved residues of the Rieske iron-sulfur subunit of the cytochrome $b c 1$ complex of Rhodobacter sphaeroides blocking or impairing quinol oxidation. Biochemistry, 32(32), 8083-8091. https://doi. org/10.1021/bi00083a005.

Wang, Y., Li, J., \& Liu, A. (2017). Oxygen activation by mononuclear nonheme iron dioxygenases involved in the degradation of aromatics. Journal of Biological Inorganic Chemistry, 22(2-3), 395-405. https://doi.org/10.1007/ s00775-017-1436-5.

Wackett, L. P., Kwart, L. D. \& Gibson, D. T. (1988). Benzylic monooxygenation catalyzed by toluene dioxygenase from Pseudomonas putida. Biochemistry, 27(4), 1360-1367.

Wolfe, M. D., Altier, D. J., Stubna, A., Popescu, C. V., Münck, E. \& Lipscomb, J. D. (2002). Benzoate 1,2-dioxygenase from Pseudomonas putida: single turnover kinetics and regulation of a two-component Rieske dioxygenase. Biochemistry, 41(30), 9611-9626. https://doi.org/10.1021/ bi025912n.

Wu, H., Tian, C., Song, X., Liu, C., Yang, D. \& Jiang, Z. (2013). Methods for the regeneration of nicotinamide coenzymes. Green Chemistry, 15(7), 1773-1789. https:// doi.org/10.1039/c3gc37129h.

Yagi, J. M. \& Madsen, E. L. (2009). Diversity, abundance, and consistency of microbial oxygenase expression and biodegradation in a shallow contaminated aquifer. Applied and Environmental Microbiology, 75(20), 6478-6487. https://doi.org/10.1128/AEM.01091-09.

Yoder, M. D., Thomas, L. M., Tremblay, J. M., Oliver, R. L., Yarbrough, L. R. \& Helmkamp, G. M. (2002). Structure of a multifunctional protein. Journal of Biological Chemistry, 276(12), 9246-9252. https://doi.org/10.1074/ jbc.m010131200.

Yoshiyama-Yanagawa, T., Enya, S., Shimada-Niwa, Y., Yaguchi, S., Haramoto, Y., Matsuya, T., Shiomi, K., Sasakura, Y., Takahashi, S.,Asashima, M., Kataoka, H.\& Niwa, R. (2011). The conserved Rieske oxygenase DAF-36/Neverland is a novel cholesterol-metabolizing enzyme. Journal of Biological Chemistry, 286(29), 25756-25762. https://doi. org/10.1074/jbc.M111.244384.

Zachos, I., Nowak, C. \& Sieber, V. (2019). Biomimetic cofactors and methods for their recycling. Current Opinion in Chemical Biology, 49, 59-66. https://doi.org/10.1016/j. cbpa.2018.10.003.

Zhu, Y., Jameson, E., Crosatti, M., Schafer, H., Rajakumar, K., Bugg, T. D. H. \& Chen, Y. (2014). Carnitine metabolism to trimethylamine by an unusual Rieske-type oxygenase from human microbiota. Proceedings of the National Academy of Sciences, 111(11), 4268-4273. https://doi.org/10.1073/ pnas. 1316569111. 